\title{
UNUSUAL LOCK-IN THERMOGRAPHY SIGNALS: SCHOTTKY-TYPE GRID CONTACTS, PELTIER EFFECTS, AND THERMAL WAVE INTERFERENCE
}

\author{
O. Breitenstein, J.P. Rakotoniaina \\ Max Planck Institute of Microstructure Physics, Weinberg 2, D-06120 Halle, Germany
}

\begin{abstract}
The technique of lock-in thermography (LIT) provides a number of well-established methods to image the lateral homogeneity of electrical parameters of solar cells. Using these methods, linear (ohmic) and nonlinear (diode-like) local leakage currents (edge currents, shunts), local Joule heating, and inhomogeneities of the series resistance and of the minority carrier lifetime can be imaged. However, in some cases, unusual LIT signals appear, which cannot be interpreted in the frame of generally accepted LIT methods. This contribution explains LIT signals connected with Schottky-type grid contacts, Peltier effects, and thermal wave interference, which is important to avoid any misinterpretation of LIT results.
\end{abstract}

\section{INTRODUCTION}

Lock-in thermography (LIT) is meanwhile standing for a whole group of solar cell and solar material characterization techniques, which are all aiming to image lateral inhomogeneities of the electronic parameters of solar cells or solar materials [1-3]. Until now, a number of assumptions were always implicitely made for interpreting LIT results, which are e.g. the assumption of good ohmic contacts both at the emitter and at the base, and the neglection of Peltier effects at these contacts. LIT experiments performed in the dark (DLIT [2]) are usually interpreted based on the energy conservation law, hence the local $\mathrm{T}$ modulation amplitude has been interpreted as a measure of the local dissipated power density. However, in some cases, certain signal features are observed in LIT investigations on solar cells, which cannot be interpreted in the frame of the pervious generally accepted models. For example, LIT experiments performed by pulsed illumination under short circuit conditions ( $\mathrm{J}_{\mathrm{sc}}$-ILIT) sometimes show bright spots below grid lines, which disappear if the measurement is performed under forward bias. It will be shown that these are due to Schottky-type grid contacts. In other cases, the structure of the ohmic base contact becomes visible in LIT images. This is often due to the Peltier effect, which appears if the current is passing between the base metal and the silicon base material. Finally, in some cases, local minima of the LIT signal may appear, which are not due to local minima of the dissipated heat, but have to be interpreted as interferences between the thermal waves originating from different heat sources in the sample. This contribution explains the physical origin of such unusual LIT signals.

\section{THE USUAL INTERPRETATION OF LIT SIGNALS}

In non-illuminated (dark) lock-in thermography (DLIT) the amplitude signal is a measure of the locally dissipated power density [1]. Hence, a homogeneous bright region usually indicates homogeneous carrier injection, and bright lines or spots are indicating shunts or Joule heating. In illuminated LIT (ILIT) performed by pulsed illumination, the whole area of a solar cell appears bright because of the essentially homogeneous carrier thermalization [2]. If this measurement is performed under short circuit conditions $\left(\mathrm{J}_{\mathrm{sc}}-\mathrm{ILIT}\right)$, dark regions are indicating noncontacted regions, and even brighter regions are indicating Joule losses due to horizontal current flow [2]. Also strong shunts may appear in $\mathrm{J}_{\mathrm{sc}}$-ILIT as bright spots, since the local bias is never exactly zero. However, bright spots, which are not visible in DLIT, are not expected to appear in $\mathrm{J}_{\mathrm{sc}}$-ILIT. If LIT is performed under pulsed illumination, and the bias is also pulsed to maximum power point conditions (mpp-ILIT), all energy losses in operation are visible. Hence here a bright region is caused by carrier thermalization losses or homogeneous injection, and bright spots or lines are caused by shunts or Joule heat. $\mathrm{R}_{\mathrm{s}}$ IILIT means that the illumination is performed continuously and the bias is pulsed between short circuit and mpp (usually close to $0.5 \mathrm{~V}$ ). In this operation mode, well-contacted regions in a cell are appearing dark, and non-contacted regions and shunts are appearing bright. Dark spots are not expected to appear in $\mathrm{R}_{\mathrm{s}}$ ILIT.

\section{SCHOTTKY-TYPE GRID CONTACTS}

The emitter of silicon solar cells is highly doped so that the metallic (usually silver) grid lines yield a good ohmic contact. However, if the emitter doping concentration should be too low, or if the grid firing conditions should be inappropriate, the contact resistance may be too high, leading to an increased series resistance, which degrades the solar cell parameters. Such poorly contacted regions can easily be imaged by methods like Corescan, CELLO, $\mathrm{R}_{\mathrm{s}}$-ILIT or $\mathrm{J}_{\mathrm{sc}}$-ILIT [3]. Regions of high series resistance appear dark in $\mathrm{J}_{\mathrm{sc}}$-ILIT and bright in $\mathrm{R}_{\mathrm{s}}$-ILIT. However, in some cases bright spots are appearing in $\mathrm{J}_{\mathrm{sc}}$-ILIT, corresponding to dark spots in $\mathrm{R}_{\mathrm{s}}$-ILIT, which are definitely no regions of especially low series resistance. As Fig. 1 shows, these spots are vertically arranged, hence they are lying in positions of grid lines, and they are visible neither in dark lock-in thermography (DLIT) nor in mpp-ILIT. These measurements were performed at a relatively high lock-in frequency of $48 \mathrm{~Hz}$, leading to a good spatial 
resolution [1]. Indeed, the left part of the sample, where these unusual contrast features appear, is a noncontacted region [3]. Our physical interpretation of this phenomenon is given in Fig. 2. Obviously, in the positions of these spots there are Schottky contacts between the grid metal and the emitter. If the grid is at base potential (short cicuit condition, see Fig. 2 a), in the non-contacted regions the photocurrent leads to an increase of the emitter potential until a forward current is flowing across the Schottky contacts, leading to the observed bright spots. Note that these Schottky junctions show a lower barrier height than the pn-junction. If a forward bias is applied to the cell (Fig. 2 b), both without illumination (DLIT) and under illumination (mpp-ILIT), the Schottky diodes become essentially unbiased or even reversebiased, hence the bright spots disappear.
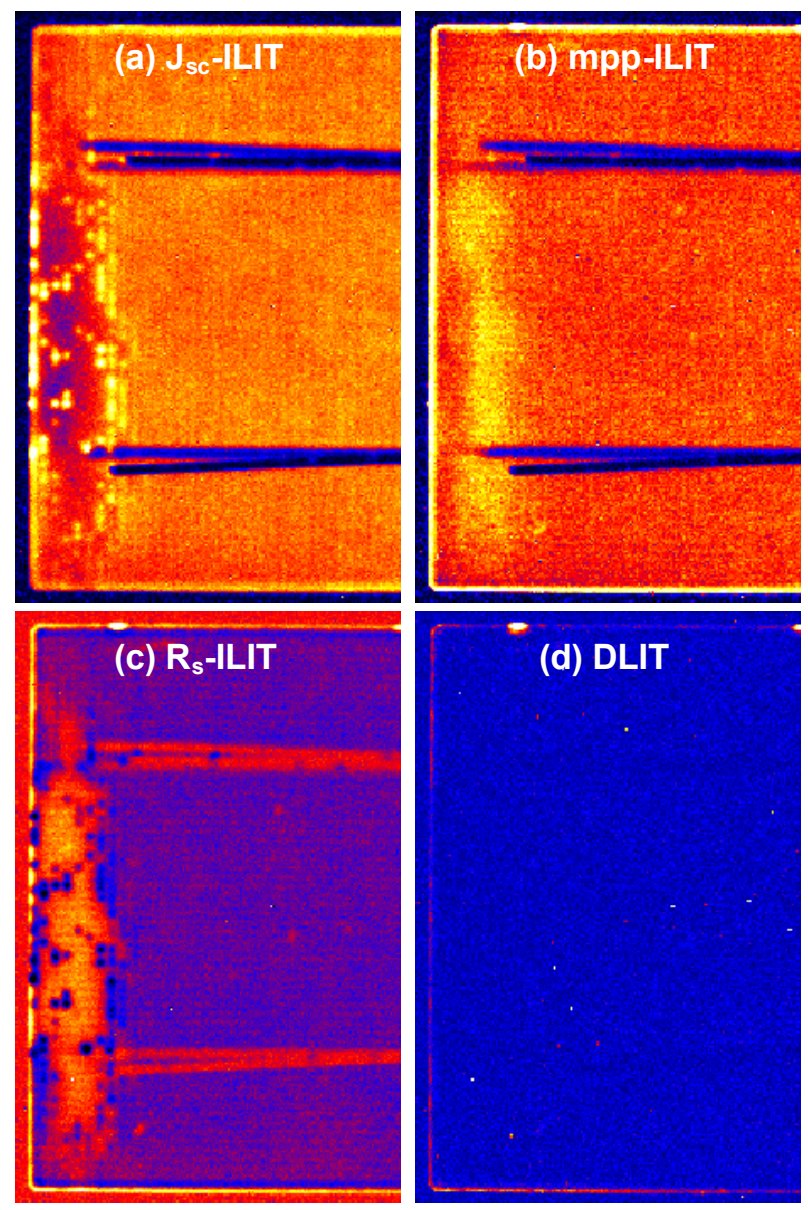

Fig. 1: $\mathrm{J}_{\mathrm{sc}}$-ILIT image (a), mpp-ILIT image (b), $\mathrm{R}_{\mathrm{s}}$-ILIT image (c), and $0.5 \mathrm{~V}$ DLIT image (d) of a region in a monocrystalline solar cell showing Schottky diodes in a non-contacted region

\section{PELTIER EFFECTS}

It has been shown that the heat dissipated in a solar cell is the sum of different heating contributions (thermalization, recombination, and Peltier heating) and different Peltier cooling contributions [2, 3]. The strongest
Peltier cooling effect is that appearing at the pn-junction, if a forward current is injected from the emitter into the base. This effect cannot be observed separately, since it is always spatially connected with minority carrier recombination heat in the bulk. The second largest Peltier cooling effect appears at the base contact of the cell, if a forward current is flowing from the base metal into the base. If the corresponding current density is low, and if carrier recombination appears in the position of the base contact current flow, this cooling action is superimposed to recombination heat, hence it is also not separately observable.

a)

$$
\text { forward biased illuminated }
$$
Schottky diode pn junction

b)

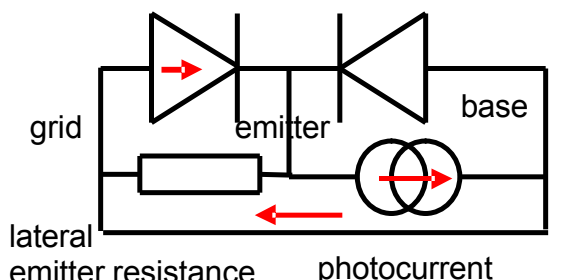
unbiased forward biased Schottky diode pnjunction

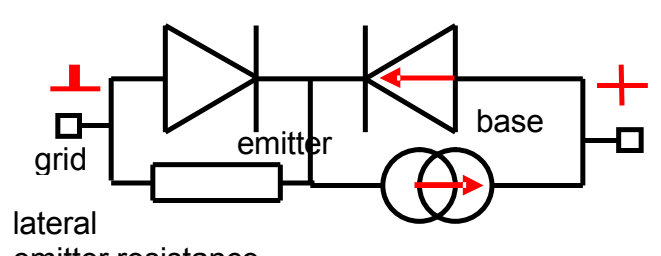

emitter resistance

photocurrent

Fig. 2: Electronic equivalent circuit of an illuminated noncontacted region in a solar cell showing an emitter Schottky diode, under short circuit (a) and under mppcondition (b)

Only if the base contact current flows in another lateral position as recombination takes place, the Peltier effect can be observed separately. This is the case e.g. for monolithic integrated "high-voltage cells" in so-called "frame" geometry [4]. Here, each single cell is weakly shunted left and right by a stripe of silicon material. For keeping the conductivity across this stripe at a minimum, the metallic base contacts are ending at both sides of this stripe. If a small bias is applied to the device, left and right of each single cell a current flows from the base metallization into this silicon stripe, and from the stripe back to the base metallization of the next cell. At the edge of the metallization, this contact current shows a local maximum, leading to an observable Peltier effect. The technique to separately display resistive (Joule) heat and the Peltier effect is to make measurements under both polarities of the bias. Then, the sum of both $-90^{\circ}$ thermograms displays the resistive heat, whereas the difference displays the Peltier heat of cooling separately ([1], see Fig. 3). The $-90^{\circ}$ signal has to be used here, 
since the amplitude signal does not allow to distinguish between positive and negative signals.

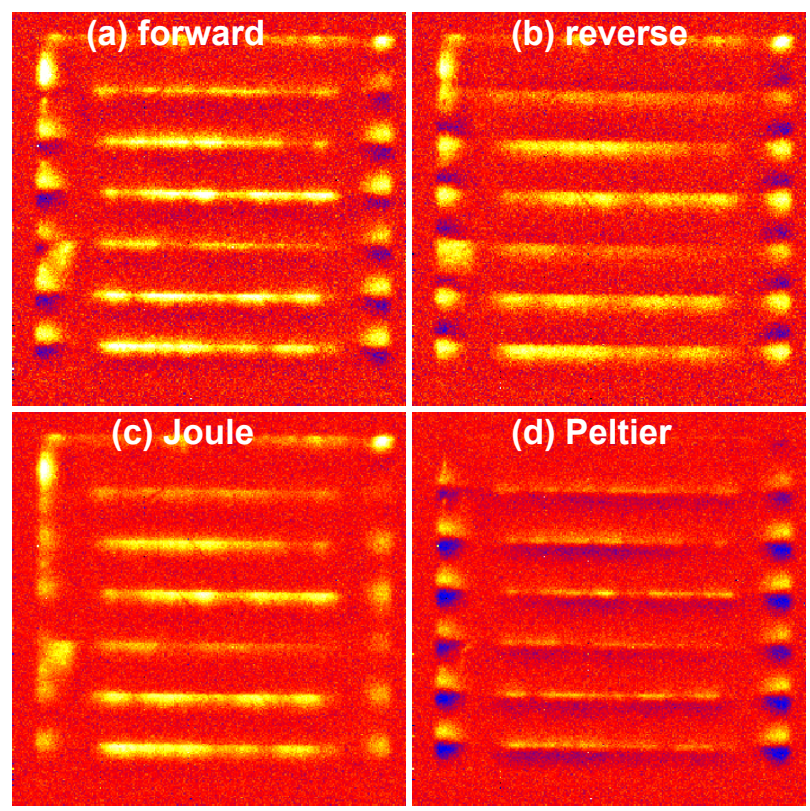

Fig. 3: Lock-in thermograms of a high voltage cell [4], taken under low forward (a) and reverse bias (b). Image c): Resistive (Joule) contribution, obtained by adding up (a) and (b), and image d): Peltier contribution, obtained by subtracting (a) and (b) [2] (dark = cooling, bright = heating).

\section{THERMAL WAVE INTERFERENCE}

As a rule, the LIT amplitude signal is considered as a local measure of the dissipated power. However, in a LIT experiment each local heat source generates thermal waves, which may interfere with each other. Note that thermal waves are inherently strongly damped. The amplitude of a plane thermal wave decays within one period by a factor of $\exp (-2 \pi)=1 / 535$ [1]. Hence, the usuable "coherence length" of thermal wave is only a small fraction of the wavelength, so that interference is quite improbable. Nevertheless, it can be observed if a strong local signal (e.g. a line shunt) is embedded in a weak homogeneous heat source, like a horizontal current flowing in the emitter. Fig. 4 shows the amplitude (a) and the phase image (b) of such a configuration measured at a lock-in frequency of $125 \mathrm{~Hz}$. The extended bright areas around the line shunts are essentially homogeneously heated regions due to lateral current flowing in the emitter, but the local minima of the signal around each line shunt are due to interference. In this region, the phase signal varies monotonically, proving the interference nature of this phenomenon. Note that in this case the cell was imaged without covering it by a black IR emitter foil [1]. Therefore the IR signal was mainly emitted by the AI back contact region, which is the usual case if uncovered solar cells are imaged by LIT.

\section{CONCLUSIONS}

As in any other physical investigation method, the interpretation of lock-in thermography (LIT) experiments requires fundamental physical knowledge. Without knowing the physical processes appearing during the measurement in detail, there is the danger for misinterpreting the results.
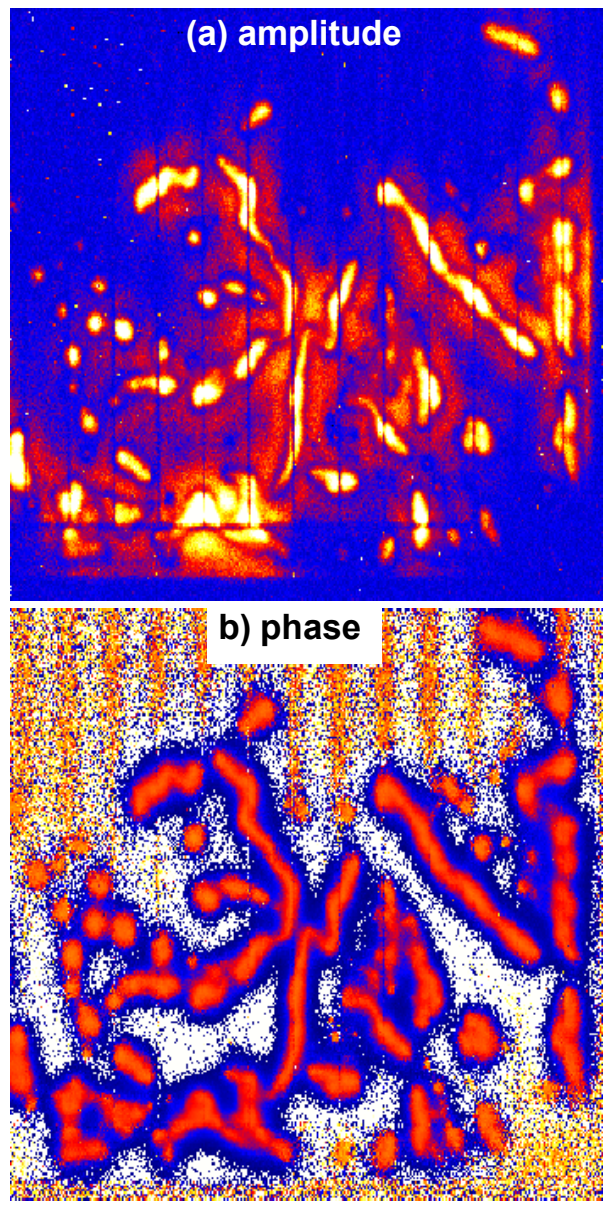

Fig. 4: DLIT amplitude image (a) and phase image (b) of a region in a solar cell showing line-shaped shunts and homogeneous heating due to a horizontal current flowing in the emitter

In this contribution we have introduced three different mechanisms, which may become visible if some assumptions commonly made for LIT are not met. If the grid contact is not an ohmic one, as is usually assumed, local Schottky diodes between grid metal and emitter layer may lead to the described unusual LIT signals. If only one of the different LIT experiments shown in Fig. 1 would be made, the unusual nature of theses signals maybe would not become obvious. So this investigation shows the importance to make both ILIT and DLIT investigations on one and the same sample. For a solar cell with the usual large-area base contact, the Peltier effect for the current flow between this contact and the base is usually 
negligible. However, if the cell is imaged e.g. at an unusually low bias as for Fig. 3, Peltier effects may become the dominating heat source. This contribution has shown that it is advantageous to use the $-90^{\circ}$ signal to detect Peltier signals, and how a Peltier signal can be separated from other resistive or recombination-induced heat sources by inversing the polarity and adding and subtracting the correspondent images. Another basic assumption of all thermographic techniques is that the thermal signal is proportional to the local dissipated power density. Of course, this holds only within the limit of the spatial resolution, which for lock-in thermography is given by the thermal diffusion length [1], usually being in the order of $1 \mathrm{~mm}$. Since all heat sources are pulsed in-phase, and heat diffusion occures via thermal waves, also interference effects should be considered. As described in the previous section, these effects are usually negligible, but in some cases they may appear. Another effect, which was not mentioned here but in other publications [1] is the usually inhomogeneous IR emissivity of the sample, which also may be misinterpreted as an inhomogeneity of the local heat dissipation density. We hope that this contribution helps to avoid misinterpretation of LIT results.

The authors are grateful to S. Keller and W. Neu (University of Konstanz) for providing the high-voltage cells used for demonstrating the Peltier effect, and to A.S.H. van der Heide (ECN Petten) for providing the cell used for demonstrating the Schottky diode signal. This work was supported by the German BMU under contract No. 0329846D.

\section{REFERENCES}

[1] O. Breitenstein, M. Langenkamp, "Lock-in Thermography - Basics and Use for Functional Diagnostics of Electronic Components", Springer (Heidelberg) 2003.

[2] O. Breitenstein, J.P. Rakotoniaina, G. Hahn, M. Kaes, T. Pernau, S. Seren, W. Warta, J. Isenberg, "Lock-in Thermography - A Universal Tool for Local Analysis of Solar Cells", Proc. 20th European Photovoltaic Solar Energy Conference and Exhibition, Barcelona, 6-10 June 2005, pp. 590-593

[3] O. Breitenstein, J.P. Rakotoniaina, A.S.H. van der Heide, J. Carstensen, "Series Resistance Imaging in Solar Cells by Lock-in Thermography", Progr. Photovolt.: Res. Appl. 13 (2005) 645-660.

[4] S. Keller, W. Neu, P. Fath, E. Bucher, "Quantitative Comparison of Different Geometries for HighVo Solar cells", Proc. 17th Eur. PVSEC, Munich 2001, pp. 17351738. 